\title{
EXISTENCE OF PERFECT PICARD SETS
}

\author{
KIKUJI MATSUMOTO
}

Dedicated to the memory of Professor TADASI NAKayama

1. Let $E$ be a totally disconnected compact set in the $z$-plane and let $\Omega$ be its complement with respect to the extended $z$-plane. Then $\Omega$ is a domain and we can consider a single-valued meromorphic function $f(z)$ in $\Omega$ which has a transcendental singularity at each point $\zeta \in E$. Suppose that $E$ is a null-set of the class $W$ in the sense of Kametani [4] (= the class $N_{\mathfrak{B}}$ in the sense of Ahlfors and Beurling [1]). Then the cluster set of $f(z)$ at each transcendental singularity is the whole $w$-plane and hence $f(z)$ has an essential singularity at each point of $E$. We shall say that a value $w$ is exceptional for $f(z)$ at an essential singularity $\zeta \in E$ if there exists a neighborhood of $\zeta$ where the function $f(z)$ does not take this value $w$. If each $f(z)$ has at most $n$ exceptional values at each singularity $\zeta \in E$, we shall call $E$ an $n$-Picard set using the terminology of Lehto [5] and call a 2-Picard set a Picard set simply. For any $E$, by Besse's theorem, there exists a single-valued regular function $g(z)$ in $\Omega$ possessing $E$ as the set of singularities. Therefore, considering the function $\exp g(z)$ in $\Omega$, we see that there exists no 1 -Picard set. Thus we need consider $n$-Picard sets only for $n \geqq 2$.

For any countable $E$, every $f(z)$ has at most two exceptional values at each singularity $\zeta \in E$, because any neighborhood of $\zeta$ contains isolated points of $E$, and hence $E$ is a Picard set. But for a non-countable $E$, there needs some condition in order to be an $n$-Picard set for some $n$, even if $E$ is of logarithmic capacity zero (see Matsumoto [6]). Carleson [3] and the author [7], [8] have given sufficient conditions for sets $E$ to be $n$-Picard sets for $n$ not smaller than 3 and examples of perfect $E$ by means of Cantor sets. There has remained a very interesting problem unsolved. Is there a perfect Picard set?

The purpose of this paper is to give Cantor sets which are Picard sets. The Schottky theorem will also play important roles as in papers [3], [7], [8].

Received May 26, 1965. 
2. We shall consider the Riemann sphere $\Sigma$ with radius $1 / 2$ touching the $w$-plane at the origin. For any two points $w$ and $w^{\prime}$ in the $w$-plane we denote by $\left[w, w^{\prime}\right]$ the chordal distance between them, that is,

$$
\left[w, w^{\prime}\right]= \begin{cases}\frac{\left|w-w^{\prime}\right|}{\sqrt{\left(1+|w|^{2}\right)\left(1+\left|w^{\prime}\right|^{2}\right)}} & \text { if } w \neq \infty \text { and } w^{\prime} \neq \infty \\ \frac{1}{\sqrt{1+|w|^{2}}} & \text { if } w^{\prime}=\infty .\end{cases}
$$

Further we denote by $C(w ; \delta)(\delta>0)$ the spherical open disc with center $w$ and with chordal radius $\delta$.

First we shall prove the following lemma which is a revised form of Carleson's [3].

Lемма 1. Let $w=f(z)$ be a single-valued meromorphic function on an annulus $1 \leqq|z| \leqq e^{\mu}(\mu>0)$. If $f(z)$ takes there no value in a spherical disc $C\left(w_{0} ; \delta\right)$, then there exists a positive constant $A_{\delta}$ depending only on $\delta$ such that the diameter of the image of $|z|=e^{\mu / 2}$ by $f(z)$ with respect to the chordal distance is dominated by $A_{\delta} e^{-\mu / 2}$ for sufficiently large $\mu$.

In particular, if $\delta$ is sufficiently close to 1 , that is, the complementary spherical disc $C\left(-1 / \bar{w}_{0} ; d\right)$ of $C\left(w_{0} ; \delta\right), d=\sqrt{1-\delta^{2}}$, has a radius sufficiently small, we have

$$
A_{\delta}<B d
$$

where $B$ is a positive constant.

Proof. We may assume without any loss of generality that the center $w_{0}$ of $C\left(w_{0} ; \delta\right)$ is the point at infinity, for otherwise we can transform $w_{0}$ to the point at infinity by the linear transformation $\left(1+\bar{w}_{0} w\right) /\left(w-w_{0}\right)$, under which the chordal distance remains invariant. Let $|w|>M$ be the domain in the $w$ plane corresponding to $C\left(w_{0} ; \delta\right)$. Then

$$
|f(z)| \leqq M \quad \text { on } \quad 1 \leqq|z| \leqq e^{u} .
$$

By Cauchy's integral theorem, we have

$$
f^{\prime}(z)=\frac{1}{2 \pi i}\left\{\int_{|\zeta|=e^{i}} \frac{f(\zeta)}{(\zeta-z)^{2}} d \zeta-\int_{|\zeta|=1} \frac{f(\zeta)}{(\zeta-z)^{2}} d \zeta\right\}
$$

for every $z$ on $|z|=e^{\mu / 2}$ and hence, if $\mu \geqq 2$, 


$$
\left|f^{\prime}(z)\right| \leqq \frac{M}{2 \pi}\left\{\frac{2 \pi e^{\mu}}{\left(e^{\mu}-e^{\mu / 2}\right)^{2}}+\frac{2 \pi}{\left(e^{\mu / 2}-1\right)^{2}}\right\} \leqq \frac{2 e^{2}}{(e-1)^{2}} M e^{-\mu} .
$$

Therefore we have

$$
\int_{|z|=e^{\mu / 2}}\left|f^{\prime}(z) \| d z\right| \leqq \frac{2 e^{2}}{(e-1)^{2}} M e^{-\mu} \cdot 2 \pi e^{\mu / 2}=\frac{4 \pi e^{2}}{(e-1)^{2}} M \cdot e^{-\mu / 2} .
$$

The left side is the length of the image curve $f\left(|z|=e^{\mu / 2}\right)$, and hence the diameter of the image of $|z|=e^{u / 2}$ by $f(z)$ with respect to the metric $|d w|$, consequently with respect to the chordal distance, is dominated by $\left(2 \pi e^{2} /(e-1)^{2}\right) M e^{-\mu / 2}$. We can take $\left(2 \pi e^{2} /(e-1)^{2}\right) M$ as $A_{\delta}$, for $M$ depends only on $\delta$.

If $d<1 / 2$, then $M<2 d$. Hence

$$
B=4 \pi e^{2} /(e-1)^{2}
$$

is one of the wanted. Our lemma is established.

Now let $w=f(z)$ be a single-valued regular function in an annulus $1<|z|<e^{u}$ $(\mu>0)$ omitting two values 0 and 1 . We use Bohr-Landau's theorem [2]; if $g(z)$ is regular in $|z|<1$ and $g(z) \neq 0,1$ there, then

$$
\max _{|z|=r}|g(z)| \leqq \exp \left(\frac{K \log (|g(0)|+2)}{1-r}\right) \quad \text { for any } r, 0 \leqq r<1,
$$

where $K$ is a positive constant (a precise form of Schottky's theorem). From this we can prove the following corollary of Lemma 1.

Corollary. There exists a positive constant $A$ not depending on $\mu$ and $f(z)$ such that the diameter of the image of $|z|=e^{\mu / 2}$ by $f(z)$ with respect to the chordal distance is dominated by A $e^{-\mu / 2}$ for sufficiently large $\mu$.

Proof. From Bohr-Landau's theorem, we can see easily that if $w=g(z)$ is a regular function in $1<|\boldsymbol{z}|<\boldsymbol{e}^{\sigma}(\sigma>0)$ such that

$$
g(z) \neq 0,1 \quad \text { and } \quad \min _{|z|=e^{0 / 2}}|g(z)|<a \quad \text { for a positive } a,
$$

then there is a positive constant $b$ depending only on $a$ and $\sigma$ such that

$$
\max _{z \mid=e^{\sigma / 2}}|g(z)| \leqq b .
$$

For a fixed $\sigma>0$, we shall show, supposing $\mu>\sigma$, that there exists a positive number $\delta$ not depending on $\mu$ and $f(z)$ such that the image curves of $|z|=e^{\sigma / 2}$ and $|z|=e^{\mu-\sigma / 2}$ by $f(z)$ lie outside at least one of three discs $C(0 ; \delta), C(1 ; \delta)$ 
and $C(\infty ; \delta)$. In fact, let $z_{1}$ and $z_{2}$ be points on $|z|=e^{\sigma / 2}$ and $|z|=e^{\mu-\sigma / 2}$ respectively. Then $f\left(z_{1}\right)$ and $f\left(z_{2}\right)$ lie outside at least one of three discs $C\left(0 ; \delta^{\prime}\right)$, $C\left(1 ; \delta^{\prime}\right)$ and $C\left(\infty ; \delta^{\prime}\right)$, where $\delta^{\prime}$ is a positive number such that these three discs are mutually disjoint and hence can be taken independently of $\mu$ and $f(z)$. Suppose that $f\left(z_{1}\right)$ and $f\left(z_{2}\right)$ lie outside $C\left(\infty ; \delta^{\prime}\right)$. Then by the fact mentioned above, we can find a positive $\delta_{\infty}$ such that the image curves of $|z|=e^{\pi / 2}$ and $|z|=e^{\mu-\sigma / 2}$ by $f(z)$ lie outside $C\left(\infty ; \delta_{\infty}\right)$. Next suppose that $f\left(z_{1}\right)$ and $f\left(z_{2}\right)$ lie outside $C\left(1 ; \delta^{\prime}\right)$. Then we see using the linear transformation which transforms points $w=0, w=1$ and $w=\infty$ to points $w=1, w=\infty$ and $w=0$ respectively that there is a positive $\delta_{1}$ such that the images of $|z|=e^{\pi / 2}$ and $|z|=e^{\mu-\pi / 2}$ by $f(z)$ lie outside $C\left(1 ; \delta_{1}\right)$. Similarly we can find a positive $\delta_{0}$ and set

$$
\delta=\min \left\{\delta_{0}, \delta_{1}, \delta_{\infty}\right\}>0 .
$$

Obviously this $\delta$ satisfies our conditions. Now by the maximum principle we see that the image of $e^{\sigma / 2} \leqq|z| \leqq e^{\mu-\sigma / 2}$ by $f(z)$ lies outside at least one of $C(0 ; \delta), C(1 ; \delta)$ and $C(\infty ; \delta)$. Hence by Lemma 1 we can conclude that the diameter of the image of $|z|=e^{\mu / 2}$ by $f(z)$ with respect to the chordal distance is dominated by $A_{\delta} e^{-(\mu-\sigma) / 2}$, so that $A=A_{\delta} e^{\sigma / 2}$ satisfies our condition.

3. Let $E$ be a Cantor set on the closed interval $I_{0}:[-1 / 2,1 / 2]$ on the real axis of the $z$-plane with successive ratios $\xi_{n}, 0<\xi_{n}=2 \ell_{n}<2 / 3$. Defining the Cantor set $E$, we repeat successively to exclude an open segment from the middle of another segment and there remain $2^{n}$ segments of equal length $\Pi_{k=1}^{n} \ell_{k}$ after we repeat $n$ times, beginning with the interval $I_{0}$. We denote these segments by $I_{n, k}\left(n=1,2, \ldots ; k=1,2, \ldots, 2^{n}\right)$ and denote by $S_{n, k}$ $\left(n=1,2, \ldots ; k=1,2, \ldots, 2^{n}\right)$ the following annuli on the complementary domain $\Omega$ of $E$ :

$$
S_{n, k}=\left\{z ;\left(\prod_{k=1}^{n} \ell_{k}\right)\left(1-\ell_{n+1}\right)<\left|z-z_{n, k}\right|<\left(\Pi_{k=1}^{n-1} \ell_{k}\right)\left(1-\ell_{n}\right) / 2\right\},
$$

where $z_{n, k}$ is the middle point of $I_{n, k}$. The harmonic modulus $\mu_{n}$ of $S_{n, k}$ is greater than $\log \left(2 / 3 \xi_{n}\right)$. We map $S_{n, k}$ conformally onto the annulus $1<|\eta|<e^{\mu_{n}}$ and consider the inverse image $\Gamma_{n, k}$ of the circle $|\eta|=e^{\mu_{n} / 2}$ on $S_{n, k}$. Supposing that $S_{n, k}$ encloses $S_{n+1,2 k-1}$ and $S_{n+1,2 k}$, we denote by $\Delta_{n, k}$ the triply connected domain bounded by three curves $\Gamma_{n, k}, \Gamma_{n+1,2 k-1}$ and $\Gamma_{n+1,2 k}$. We now prove the following 
Lemma 2. Let the successive ratios $\xi_{n}$ satisfy the condition

$$
\lim _{n \rightarrow \infty} \xi_{n}=0
$$

and let $w=f(z)$ be a single-valued regular function in $\Omega$ which omits two values 0 and 1 and has $E$ as the set of essential singularities. Then for any sufficiently small $\delta<0$, there exists an infinite number of $\Delta_{n, k}$ such that the images of the three boundary components $\Gamma_{n, k}, \Gamma_{n+1,2 k-1}$ and $\Gamma_{n+1,2 k}$ are contained completely in the three discs $C(0 ; \delta), C(1 ; \delta)$ and $C(\infty ; \delta)$ one by one, where we assume that these three discs are mutually disjoint, and $f(z)$ takes each value outside the union of these three discs once and only once in $\Delta_{n, k}$.

Proof. Contrary suppose that there exists only a finite number of $\Delta_{n, k}$ such that their three boundary components are mapped into the three discs $C(0 ; \delta), C(1 ; \delta)$ and $C(\infty ; \delta)$ one by one, and denote by $n_{0}$ the maximum of $\boldsymbol{n}$ taken over all such $\Delta_{n, k}$. Since

$$
\mu_{n}>\log \left(2 / 3 \xi_{n}\right) \quad \text { and } \quad \lim _{n \rightarrow \infty} \xi_{n}=0
$$

we can take $n_{1} \geqq n_{0}$, for a fixed $\sigma>0$, so large that for each $n \geqq n_{1}$

$$
\mu_{n}>2+\sigma, A e^{-\mu_{n} / 2}<K=\min \{1 / 24, \delta / 3\} \quad \text { and } B e^{-\mu_{n} / 2}<1 / 12,
$$

where $A$ and $B$ are the constants in Lemma 1 and its corollary. The diameter of the image of $\Gamma_{n, k}$ with respect to the chordal distance is dominated by $A e^{-\mu_{n} / 2}$, consequently by $K$, if $n \geqq n_{1}$, and hence there exists a spherical disc $C_{n, k}$ with chordal radius $K$ which contains completely the image $f\left(\Gamma_{n, k}\right)$. For $n \geqq n_{1}$ take $\Delta_{n, k}$ with boundary curves $\Gamma_{n, k}, \Gamma_{n+1,2 k-1}$ and $\Gamma_{n+1,2 k}$. Then, since $K<\delta / 3$, at least one of $C(0 ; \delta / 3), C(1 ; \delta / 3)$ and $C(\infty ; \delta / 3)$, say $C(\infty ; \delta / 3)$, is disjoint from the union of $C_{n, k}, C_{n+1,2 k-1}$ and $C_{n+1,2 k}$ and hence each one of $C_{n, k}, C_{n+1,2 k-1}$ and $C_{n+1,2 k}$ cannot be disjoint from the union of the other two, for, if so for some one, there is $z_{0} \in \Delta_{n, k}$ such that $f\left(z_{0}\right)$ lies outside the union of $C_{n, k}, C_{n+1,2 k-1}$ and $C_{n+1,2 k}$ and can be joined to the point at infinity with a curve $A$ lying outside this union, and we are led to a contradiction that the element of the inverse function $f^{-1}$ corresponding to $z_{0}$ can be continued analytically along $A$ up to a point arbitrarily near the point at infinity so that $f(z)$ takes the value $\infty$ in $\Delta_{n, k}$. Therefore we can conclude that

$\left(1^{\circ}\right)$ for every $\Delta_{n, k}, n \geqq n_{1}$, there is a spherical disc with chordal radius 
$3 K$ containing completely the image $f\left(\Delta_{n, k}\right)$.

Next we shall consider $I_{n, k}$ for $n \geqq n_{1}+1$. Then $\Delta_{n, k}$ and some $\Delta_{n-1, k}$, have $\Gamma_{n, k}$ as the common boundary and $\Delta_{n-1, k} \cup \Gamma_{n, k} \cup \Delta_{n, k} \supset S_{n, k}$. From ( $\left.1^{\circ}\right)$, the image of $\Delta_{n-1, k} \cup \Gamma_{n, k} \cup \Delta_{n, k}$, consequently the image of $S_{n, k}$, are contained in a spherical disc with chordal radius $6 K<1 / 2$, so that, applying Lemma 1 in $S_{n, k}$ for $d=6 K$, we see that the diameter of $f\left(\Gamma_{n, k}\right)<6 K B e^{-\mu_{n} / 2}<K / 2$. Hence for $n \geqq n_{1}+1$, each boundary component of $\Delta_{n, k}$ has the image with diameter less than $K / 2$. From the same reason as above we now conclude that

$\left(2^{\circ}\right)$ for $n \geqq n_{1}+1$, the image of any $\Delta_{n, k}$ is contained in a spherical disc with chordal radius $3 K / 2$.

By induction we also see for every $p \geqq 1$ that

$\left(p^{\circ}\right)$ for $n \geqq n_{1}+p-1$, the image of any $\Delta_{n, k}$ is contained in a spherical disc with chordal radius $3 K / 2^{p-1}$.

Let $\Omega_{n, k}$ be the part of $\Omega$ bounded by the simple closed curve $\Gamma_{n, k}$ and let $z_{0}$ be a point of $\Gamma_{n, k}$. Then for any $z \in \Omega_{n, k}$, there is a $\Delta_{n+p, q}$ whose closure contains $z$ and we can find a chain $\left\{\Delta_{n+i, j(i)}\right\}(i=0,1, \ldots, p ; j(0)=k, j(p)=q)$ joining $\Delta_{n, k}$ and $\Delta_{n+p, q}$. Supposing that $n \geqq n_{1}$, we have by $\left(p^{\circ}\right)$ obtained above

$$
\begin{aligned}
\left.L f\left(z_{0}\right), f(z)\right] & \leqq \sum_{i=0}^{p} \text { diam. of } f\left(\Delta_{n+i, j(i)}\right) \text { w.r.t. the chordal distance } \\
& \leqq 2 \sum_{i=0}^{p} 3 K / 2^{i} \leqq 12 K<1 / 2 .
\end{aligned}
$$

By means of a linear transformation we can consider from the above that $f(z)$ is bounded in $\Omega_{n, k}$. On the other hand, $E$ is a linear set of linear measure zero, so that $E$ is a null-set of the class $W$ (Kametani [4], Ahlfors and Beurling [1]). Hence each point of the part of $E$ contained in the interior of $\Gamma_{n, k}$ must be a removable singularity of the bounded function $f(z)$; this contradicts our assumption that each point of $E$ is an essential singularity of $f(z)$.

Next suppose that $f(z)$ takes a value $w_{0}$ outside the union of $C(0 ; \delta)$, $C(1 ; \delta)$ and $C(\infty ; \delta)$ at two points $z^{\prime}$ and $z^{\prime \prime}$ in $\Delta_{n, k}$, whose boundary components $\Gamma_{n, k}, \Gamma_{n+1,2 k-1}$ and $\Gamma_{n+1,2}$ are mapped into the discs $C(0 ; \delta), C(1 ; \delta)$ and $C(\infty ; \delta)$ one by one, and join $w_{0}$ to $C(0 ; \delta)$ and $C(1 ; \delta)$ with curves $A^{\prime}$ and $A^{\prime \prime}$ respectively, which lie outside the union, do not intersect each other except at $w_{0}$ and do not pass through any projection of branch points of the 
Riemannian image of $\Delta_{n, k}$ by $f(z)$. The elements of the inverse function $f^{-1}$ corresponding to $z^{\prime}$ and $z^{\prime \prime}$ can be continued analytically along these curves to their end points and further from them along radii of $C(0 ; \delta)$ and $C(1 ; \delta)$ so that the curves in $\Delta_{n, k}$ corresponding to these continuations join each of $z^{\prime}$ and $z^{\prime \prime}$ to $\Gamma_{n+1,2 k-1}$ and $\Gamma_{n+1,2 k}$, where we assume that the images $\Gamma_{n+1,2 k-1}$ and $\Gamma_{n+1,2 k}$ are contained in $C(0 ; \delta)$ and $C(1 ; \delta)$ respectively, and bound with parts of $\Gamma_{n+1,2 k-1}$ and $\Gamma_{n+1,2 k}$ a domain not containing $\Gamma_{n, k}$. Since $\Delta_{n . k}$ has no boundary other than $\Gamma_{n, k}, \Gamma_{n+1,2 k-1}$ and $\Gamma_{n+1,2 k}$, this domain must be a subdomain of $\Delta_{n, k}$ and $f(z)$ must take the value $\infty$ there; this is a contradiction. Our proof is now complete.

4. We note that for each $n$ and $k\left(n=1,2, \ldots ; k=1,2, \ldots, 2^{n}\right) \Delta_{n, k}$ is bounded by $\Gamma_{n, k}, \Gamma_{n+1,2 k-1}$ and $\Gamma_{n+1,2 k}$ and lies on the right-side of $\Delta_{n, k^{\prime}}$, $1 \leqq k^{\prime}<k$. We now estimate the harmonic modulus of any doubly connected domain contained in $\Delta_{n, k} \cup \Gamma_{n+1,2 k-1} \cup \Delta_{n+1,2 k-1}$ such that one connected component of its complement contains the circles $\Gamma_{n, k}$ and $\Gamma_{n+1,2 k}$ and the other contains the circles $\Gamma_{n+2,4 k-3}$ and $\Gamma_{n+2,4 k-2}$. Moving this domain in parallel so that the right end point of $I_{n+1,2 k-1}$ comes to the origin, we see that the harmonic modulus of our domain is dominated by

$$
\log \Psi\left(\left(\Pi_{k=1}^{n} \ell_{k}\right)\left(1-\xi_{n+1}\right) / \Pi_{k=1}^{n+1} \ell_{k}\right)=\log \Psi\left(2\left(1-\xi_{n+1}\right) / \xi_{n+1}\right),
$$

where $\log \Psi(P / \rho) \quad(P, \rho>0)$ denotes the harmonic modulus of the normal domain of Teichmüller, the complement of the union of the two segments, $-\rho \leqq x \leqq 0$, $y=0$ and $P \leqq x \leqq+\infty, y=0$ in the $z$-plane $(z=x+i y)$. It is well-known that

$$
\Psi(P / \rho)<16 \frac{P}{\rho}+8
$$

and we obtain thus the following

Lemma 3. The harmonic modulus of any doubly connected domain considered above is dominated by $\log \left(32 / \xi_{n+1}\right)$.

5. Now we shall show the existence of perfect Picard sets. We shall prove the following

TheоRem. Let $E$ be a Cantor set on the closed interval $I_{0} ;[-1 / 2,1 / 2]$ on the real axis of the $z$-plane with successive ratios $\xi_{n}, 0<\xi_{n}<2 / 3$, If the ratios $\xi_{n}$ satisfy the condition 


$$
\xi_{n+1}=o\left(\xi_{n}^{2}\right),
$$

then $E$ is a Picard set.

Proof. We take $\delta>0$ so small that the discs $C(0 ; 2 \delta), C(1 ; 2 \delta)$ and $C(\infty ; 2 \delta)$ are mutually disjoint. Contrary to our assertion, let us suppose that there exists a single-valued meromorphic function $f(z)$ in the complementary domain $\Omega$ of $E$ which has $E$ as the set of essential singularities and has three exceptional values at an essential singularity $\zeta \in E$, where we may assume that these values are 0,1 and $\infty$. Since our argument given in the below is applicable locally, it will not bring any loss of generality if we shall give a contradiction under the stronger assumption that $f(z)$ omits the values 0,1 and $\infty$ in $\Omega$.

Let $n_{0}$ be so large that $A e^{-\mu_{n} / 2}<\delta / 2$ for any $n \geqq n_{0}$. By Lemma 2 there is a $A_{n, k}\left(n \geqq n_{0}\right)$ whose three boundary components are mapped into $C(0 ; \delta), C(1 ; \delta)$ and $C(\infty ; \delta)$ one by one, where we may assume that the boundary curve $\Gamma_{n+1,2 k-1}$ of $\Delta_{n, k}$ is mapped into $C(\infty ; \delta)$. Now we consider the quadruply connected domain $D=\Delta_{n, k} \cup \Gamma_{n+1,2 k-1} \cup \Delta_{n+1,2 k-1}$. The images of the boundary curves $\Gamma_{n+2,4 k-3}$ and $\Gamma_{n+2,4 k-2}$ of $D$ are contained in some spherical discs $C$ and $C^{\prime}$ with radius $A e^{-\mu_{n+2} / 2}<\delta / 2$ respectively, and we see that $C$ and $C^{\prime}$ are contained in $C(\infty ; 2 \delta)$. In fact, one of them, say $C$, must contain the point at infinity, for otherwise, $f(z)$ must take the value $\infty$ in $D$, and hence is contained in $C(\infty ; \delta)$. Suppose that $C^{\prime}$ is not contained in $C(\infty ; 2 \delta)$. Then there is a point $z \in \Delta_{n+1,2 k-1} \subset D$ whose image $f(z)$ lies in $C(\infty ; 2 \delta)-C(\infty ; \delta) \cup C^{\prime}$ and can be joined the origin or the point $w=1$ with a path not intersecting the image of the boundary of $\Delta_{n+1,2 k-1}$, so that $f(z)$ takes the value 0 or 1 in $\Delta_{n+1,2 k-1}$. Contradiction. Thus we can find a positive $d$ such that $d<2 \delta$ and the disc $C(\infty ; d)$ contains $C$ and $C^{\prime}$.

Next we shall prove that $f(z)$ takes each value outside the union of the three discs $C(0 ; \delta), C(1 ; \delta)$ and $C(\infty ; d)$ once and only once in $D$. By Lemma $2, f(z)$ takes each value outside the union of $C(0 ; \delta), C(1 ; \delta)$ and $C(\infty ; \delta)$ once and only once in $\Delta_{n, k}$, so that the inverse image $I$ of the circle $[w, \infty]=2 \delta$ on $\Delta_{n, k}$ is a simple closed curve and separates $\Gamma_{n, k}$ and $\Gamma_{n+1,2 k}$ from $\Gamma_{n+2,4 k-3}$ and $I_{n+2,4 k-2}$. Now suppose that $f(z)$ takes a value $w_{0}$ outside the union of $C(0 ; \delta), C(1 ; \delta)$ and $C(\infty ; d)$ at two points $z^{\prime}$ and $z^{\prime \prime}$ in $D$, and join $w_{0}$ with $C(0 ; \delta)$ with a curve $A$ which lies outside the union and does not pass through any projection of branch points of the Riemannian image of $D$ by $f(z)$. The 
elements of the inverse function $f^{-1}$ corresponding to $z^{\prime}$ and $z^{\prime \prime}$ can be continued analytically along $A$ to its end point and hence we see that every value on $A$ is taken by $f(z)$ at least two times in $D$. Therefore we can assume that $w_{0}$ lies outside $C(\infty ; 2 \delta)$. Then one of $z^{\prime}$ and $z^{\prime \prime}$ must lie in the domain $D^{\prime}$ bounded by $\Gamma, \Gamma_{n+2,4 k-3}$ and $\Gamma_{n+2,4 k-2}$ and the corresponding element of $f^{-1}$ can be continued analytically to the origin along a curve outside $C(\infty ; 2 \delta)$, so that $f(z)$ takes the value 0 in $D^{\prime}$; this contradicts our assumption.

Now we estimate $d$ from below. To this purpose we consider the annulus $R: 2<|w|<\sqrt{1-d^{2}} / d$ corresponding to the annulus $1 / \sqrt{5}>[w, \infty]>d$ on the Riemann sphere $\Sigma$, which separates $C(0 ; \delta)$ and $C(1 ; \delta)$ from $C(\infty ; d)$. As we have seen above, the Riemannian image of $D$ covers $R$ univalently, the ring domain on $D$ corresponding to $R$, which has the same harmonic modulus as $R$, separates the boundary curves $\Gamma_{n, k}$ and $\Gamma_{n+1,2 k}$ of $D$ from the boundary curves $\Gamma_{n+24 k-3}$ and $\Gamma_{n+2,4 k-2}$ of $D$. By Lemma 3 we have thus

har. mod. of $R=\log \left(\sqrt{1-d^{2}} / 2 d\right) \leqq \log \left(32 / \xi_{n+1}\right)$.

Since $d<2 \delta<\pi / 6$, we have the estimate

$$
d \geqq\left(\sqrt{1-(\pi / 6)^{2}} / 64\right) \xi_{n+1}=\frac{1}{M} \xi_{n+1} .
$$

This implies that $C^{\prime}$ must intersect the $\operatorname{disc}[w, \infty] \geqq \xi_{n+1} / M=m$. Consider the domain $\Delta_{n+2,4 k-2}$ which, with $\Delta_{n+1,2 k-1}$, has $\Gamma_{n+2,4 k-2}$ as the common boundary. The images of three boundary curves $\Gamma_{n+2,4 k-2}, \Gamma_{n+3,8 k-5}$ and $\Gamma_{n+3,8 k-4}$ of $\Delta_{n+2,4 k-2}$ are contained in some three spherical discs $C^{\prime}=C_{n+2,4 k-2}, C_{n+3,8 k-5}$ and $C_{n+3,8 k-4}$ with radii less than $A e^{-\mu_{n+2} / 2}<A^{\prime} \sqrt{\xi_{n+2}}$, respectively, where $A^{\prime}=\sqrt{3 / 2} A$. We may suppose that $n$ is so large that for each $p \geqq 1$,

$$
\left(12 A^{\prime} M\right)^{2} \xi_{n+p+1} \leqq \xi_{n+p}^{2} .
$$

Then $C_{n+2,4 k-2}$ does not contain the point at infinity, since $A^{\prime} \sqrt{\overline{\xi_{n+2}}}<m / 12$, so that any one of $C_{n+2,4 k-2}, C_{n+3,8 k-5}$ and $C_{n+3,8 k-4}$ cannot be disjoint from the union of the other two. By the same reasoning in the proof of Lemma 2 we see that the image of $\Delta_{n+2,4 k-2}$ is contained in a spherical disc with radius less than $3 A^{\prime} \sqrt{\xi_{n+2}}<m / 4$. Since this disc intersects the disc $[w, \infty] \geqq m$, it must lie outside the $\operatorname{disc} C(\infty ; m / 2)$. Next we consider the domain $\Delta_{n+3,8 k-5}$. Each image of its three boundary curves is contained in a spherical disc with radius less than 


$$
A^{\prime} \sqrt{\xi_{n+3}} \leqq \xi_{n+2} / 12 M<A^{\prime} \sqrt{\xi_{n+2}} / 12 A^{\prime} M<m / 24,
$$

where we suppose that $A^{\prime}>1 / 6 M$; this does not bring any loss of generality. The same argument shows that the image of $\Delta_{n+3,8 k-5}$ is contained in a spherical disc with radius less than $m / 8$ and lying outside the disc $C(\infty ; m / 4)$. The same holds for $\Delta_{n+3,8 k-4}$. Since, for each $p \geqq 2$,

$$
A^{\prime} \sqrt{\xi_{n+p+1}} \leqq \xi_{n+p} / 12 M<A^{\prime} \sqrt{\xi_{n+p}} / 2,
$$

we can conclude by induction that the image of the domain $\Delta_{n+p+1, q}$ lying in the interior of the simple closed curve $\Gamma_{n+2,4 k-2}$ is contained in a spherical disc with radius less than $m / 2^{p+1}$ and lying outside the disc $C\left(\infty ; m / 2^{p}\right)$. It follows that, in the interior of $\Gamma_{n+2,4 k-2}, f(z)$ takes values only in a spherical disc with radius less than

$$
\sum_{p=1}^{\infty} m / 2^{p+1}<m / 2
$$

By means of a linear transformation we can consider that $f(z)$ is bounded in the interior of $\Gamma_{n+2,4 k-2}$; this contradicts our assumption that $f(z)$ has an essential singularity at every point of $E$. Our theorem is now established.

\section{References}

[1] L. V. Ahlfors and A. Beurling: Conformal invariants and function-theoretic null-sets, Acta Math., 83 (1950), pp. 101-129.

[2] H. Bohr and E. Landau: Über das Verhalten von $\zeta(s)$ und $\zeta_{k}(s)$ in der Nähe der Geraden $\sigma=1$, Göttinger Nachr., (1910).

[ 3 ] L. Carleson: A remark on Picard's theorem, Bull. Amer. Math. Soc., 67 (1961), pp. 142-144.

[4] S. Kametani: On Hausdorff's measures and generalized capacities with some of their applications to the theory of functions, Jap. Journ. Math., 19 (1944-48), pp. 217-257.

[ 5 ] O. Lehto: A generalization of Picard's theorem, Ark. Mat., 3 nr. 45 (1958), pp. 495500.

[6] K. Matsumoto: Exceptional values of meromorphic functions in a neighborhood of the set of singularities, Journ. Sci. Hiroshima Univ., A 24 (1960), pp. 143-153.

[ 7 ] K. Matsumoto: On exceptional values of meromorphic functions with the set of singularities of capacity zero, Nagoya Math. Journ., 18 (1961), pp. 171-191.

[8] K. Matsumoto: Some notes on exceptional values of meromorphic functions, Nagoya Math. Journ., 22 (1963), pp. 189-201.

\section{Mathematical Institute}

Nagoya University 